\title{
Microwave spectroscopy measurements of the hyperfine structure in antiprotonic ${ }^{3} \mathrm{He}$
}

\author{
Susanne Friedreich • Daniel Barna • Fritz Caspers • Andreas Dax • Ryugo Hayano • \\ Masaki Hori • Dezsó Horváth • Bertalan Juhász • Takumi Kobayashi • \\ Oswald Massiczek • Anna Sótér · Koichi Todoroki • \\ Eberhard Widmann • Johann Zmeskal
}

Published online: 28 August 2012

(C) The Author(s) 2012. This article is published with open access at Springerlink.com

\begin{abstract}
We shall present here the first experimental results for microwave spectroscopy of the hyperfine structure of antiprotonic He-3 and a comparison to numerical simulations of the measurement. Due to the helium nuclear spin, antiprotonic He3 has a more complex hyperfine structure than antiprotonic $\mathrm{He}-4$ which has already been studied before. Thus a comparison between theoretical calculations and the experimental results will provide a more stringent test of the three-body quantum electrodynamics (QED) theory. The comparison of measured data to simulations allows to investigate the collisional processes between the helium atoms of the target medium and the antiprotonic helium atomcules. The collision rates can not be calculated exactly, but estimated by comparison of numeric simulations with the experimental results. Two out of four super-super-hyperfine (SSHF) transition lines of the $(n, L)=(36,34)$ state were observed. The measured frequencies of the
\end{abstract}

S. Friedreich $(\bowtie) \cdot$ B. Juhász $\cdot$ O. Massiczek $\cdot$ E. Widmann $\cdot$ J. Zmeskal Stefan Meyer Institute for Subatomic Physics,

Austrian Academy of Sciences, 1090 Vienna, Austria

e-mail: susanne.friedreich@oeaw.ac.at

D. Barna - A. Dax · R. Hayano - K. Todoroki · T. Kobayashi · M. Hori

Department of Physics, University of Tokyo, Tokyo 113-0033, Japan

D. Barna $\cdot$ D. Horváth

KFKI Research Institute for Particle and Nuclear Physics,

1525 Budapest, Hungary

F. Caspers

CERN, 1211 Geneva, Switzerland

M. Hori · A. Sótér

Max-Planck-Institut für Quantenoptik, 85748 Garching, Germany

D. Horváth

Institute of Nuclear Research of the Hungarian Academy of Sciences,

4001 Debrecen, Hungary 
individual transitions are 11.12559(14) $\mathrm{GHz}$ and $11.15839(18) \mathrm{GHz}$, less than $1 \mathrm{MHz}$ higher than the current theoretical values, but still within their estimated errors. The frequency difference between the two lines also agrees with theoretical calculations.

Keywords Antiproton - Antiprotonic helium - Hyperfine structure - Spectroscopy • Laser $\cdot$ Microwave $\cdot$ Three-body quantum electrodynamics

\section{Introduction}

Antiprotonic helium $\left(\overline{\mathrm{p}} \mathrm{He}^{+}\right)$is an exotic, metastable three-body system, consisting of one electron in the ground state, an antiproton and the helium nucleus [1-4]. It is produced when an antiproton is approaching a helium atom at its ionization energy $(24.6 \mathrm{eV})$ or below. The antiproton can thus eject one of the two electrons from the ground state, replace it and get captured. The atoms occupy circular states with $L$ close to $n$, where $L$ is the angular momentum quantum number and $n$ the principal quantum number. The electron remains in the ground state. The antiproton is, due to its high mass, most likely to be captured into states with high angular momentum, i.e. $n=n_{0} \equiv \sqrt{M^{*} / m_{\mathrm{e}}} \sim 38, M^{*}$ being the reduced mass of the system. About $97 \%$ of these exotic atoms find themselves in states dominated by Auger decay and ionize within a few nanoseconds because of the Auger excitation of the electron. Only $3 \%$ of the antiprotonic helium atoms remain in metastable, radiative decay-dominated states. In this case, the change of orbital angular momentum in the Auger transition is large and thus Auger decay is suppressed. Consequently, these states are relatively long lived, having a lifetime of about 1-2 $\mu$ s. This time window can be used to do microwave spectroscopy measurements.

\section{Hyperfine structure of antiprotonic helium}

The interaction of the magnetic moments of its constituting particles gives rise to a splitting of the $\overline{\mathrm{p}}^{3} \mathrm{He}^{+}$energy levels. The coupling of the electron spin $\mathbf{S}_{\mathrm{e}}$ and the orbital angular momentum of the antiproton $L$ leads to the primary splitting of the state into a doublet structure, referred to as hyperfine $(H F)$ splitting. The non-zero spin of the ${ }^{3} \mathrm{He}$ nucleus causes a further so-called super-hyperfine $(\mathrm{SHF})$ splitting, which can be characterized by the quantum number $\mathbf{G}=\mathbf{F}+\mathbf{S}_{\mathrm{h}}=\mathbf{L}+\mathbf{S}_{\mathrm{e}}+\mathbf{S}_{\mathrm{h}}$, where $\mathbf{S}_{\mathrm{h}}$ is the spin of the helium nucleus. This results in four SHF substates. At last, the spin-orbit interaction of the antiproton orbital angular momentum and antiproton spin $\mathbf{S}_{\overline{\mathrm{p}}}$ in combination with the contact spin-spin and the tensor spin-spin interactions between the particles result in a further splitting of the SHF substates into eight substates-as illustrated in Fig. 1-which we call super-superhyperfine $(S S H F)$ splitting. This octuplet structure can be described by the quantum number $\mathbf{J}=\mathbf{G}+\mathbf{S}_{\overline{\mathrm{p}}}=\mathbf{L}+\mathbf{S}_{\mathrm{e}}+\mathbf{S}_{\mathrm{h}}+\mathbf{S}_{\overline{\mathrm{p}}}$. Even though the magnetic moment of the antiproton is larger than that of the ${ }^{3} \mathrm{He}$ nucleus, the former has a smaller overlap with the electron cloud. Therefore it creates a smaller splitting.

Transitions between the SSHF states can be induced by a magnetic field oscillating in the microwave frequency range. Due to technical limitations of the microwave input power, only the transitions which flip the spin of the electron can be measured. 
Fig. 1 A schematic drawing of the laser-microwave-laser method. The dashed arrows indicate the laser transitions between the SHF levels of the radiative decay-dominated state $(n, L)=(36,34)$ and the Auger decay-dominated state $(n, L)=(37,33)$ of $\overline{\mathrm{p}}^{3} \mathrm{He}^{+}$. The wavy lines illustrate the microwave-induced transitions between the SSHF levels of the long-lived state
Auger decaying daughter state

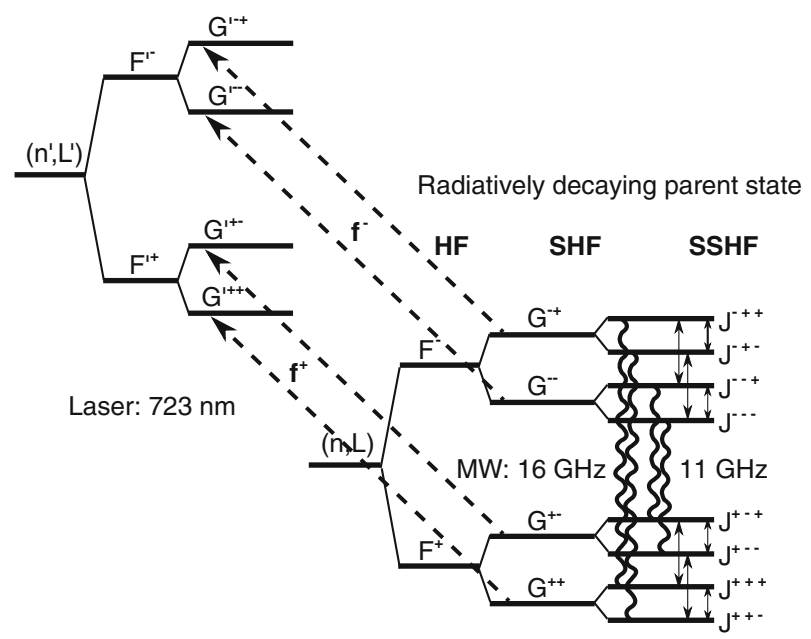

Interaction: $\quad S_{e} \quad S_{h} \quad S_{\bar{p}}$

There are four such "allowed" SSHF transitions for the $(n, L)=(36,34)$ state, of which we report on two:

$$
\begin{aligned}
& v_{\mathrm{HF}}^{--}: J^{---}=L-\frac{3}{2} \longrightarrow J^{+--}=L-\frac{1}{2} \\
& v_{\mathrm{HF}}^{-+}: J^{--+}=L-\frac{1}{2} \longrightarrow J^{+-+}=L+\frac{1}{2}
\end{aligned}
$$

\section{Laser-microwave-laser spectroscopy}

The experimental technique is a three-step process, referred to as laser-microwavelaser spectroscopy [5, 6] (Fig. 1). After antiprotonic helium is formed, the hyperfine substates are all equally populated. A short laser pulse of 10-12 ns at first induces a population asymmetry between the SSHF substates of the measured radiative decay state $(n, L)$ by transferring the majority of the antiprotons from one of the HF states of the radiative decay-dominated, metastable parent state to the Auger decaydominated, short-lived daughter state $\left(f^{+}\right.$transition in Fig. 1). The laser bandwidth of about $100 \mathrm{MHz}$ is narrow enough so that the $f^{-}$transition is not excited and the antiprotons in the other HF state are not affected. The antiprotons in the shortlived daughter state annihilate within a few nanoseconds. Subsequently, a microwave frequency pulse, tuned around the transition frequency between two SSHF $\left(\overline{\mathrm{p}}^{3} \mathrm{He}^{+}\right)$ substates of the parent state, is applied to the antiprotonic helium atoms. In practice, the microwave pulse is about $20 \mu$ s long and starts a few microseconds before the first laser pulse. This will have no effect on the population distribution as long as the laser pulse does not create an asymmetry between the SSHF substates. The length of the microwave pulse thus corresponds to the time between the end of the first and the beginning of the second laser pulse. If the microwave field is on resonance with one of the SSHF transitions, this will cause a population transfer and thus a partial 
refilling of one of the previously depopulated states. Then a second laser pulse of equal length is applied to the same transition $\left(f^{+}\right)$as before, which will again result in subsequent Auger decay of the transferred atoms and annihilation of the antiprotons. Thus the number of annihilations after the second laser pulse will be larger if more antiprotonic atoms were transferred by the microwave pulse. More details on the experimental technique and measurement parameters can be found in [7].

\section{Experimental setup}

The antiprotons for the experiment were provided by the AD (Antiproton Decelerator) at CERN [8], with a pulsed beam of $(1-3) \times 10^{7}$ antiprotons at an energy of $5.3 \mathrm{MeV}$ and a repetition rate of about $100 \mathrm{~s}$. The particles were stopped in a helium gas target, cooled down to a temperature of about $6 \mathrm{~K}$. The usual gas pressure was in the range of 150-500 mbar. The gas target was a cylindrical chamber, which also acted as a microwave resonance cavity. In order to measure the annihilation decay products two Cherenkov counters were mounted around the target volume. The resulting photons are detected by photomultipliers, which are gated off during the initial $\bar{p}$ pulse arrival [9]. The microwave pulse was synthesized by a vector network analyzer (VNA, Rhode \& Schwarz ZVB20) and amplified by a travelling wave tube amplifier (TWTA, TMD PTC6358). A waveguide system then transmitted the microwave pulse of $20 \mu$ s to the cavity. A self-developed, pulse-amplified laser system [10,11] based on a contiuous wave $(\mathrm{CW})$ laser was used to provide the two laser beams fixed to a wavelength of $723.877 \mathrm{~nm}$. The $\mathrm{CW}$ laser consists of a ring $\mathrm{Ti}$ : sapphire laser (Coherent MBR-110) pumped by a $\mathrm{Nd}: \mathrm{YVO}_{4}$ laser (Coherent Verdi) and is amplified using Bethune cells pumped by two pulsed Nd:YAG lasers (Coherent Infinity). This system allows the generation of two laser pulses with arbitrary time delay and a fluence of the two pulses in the range of $20-40 \mathrm{~J} / \mathrm{cm}^{2}$. The laser waist is $\sim 5 \mathrm{~mm}$, leading to a depletion efficiency of about $90 \%$.

The experimental method and the general design of the setup were the same as for $\overline{\mathrm{p}}^{4} \mathrm{He}^{+}[7,12]$. However, a new cryostat with a compressor-based cooling system [13] was built, which led to improvements of the operation and more efficient use of the measurement time. Out of the four allowed SSHF transitions of the state $(n, L)=$ $(36,34)$ of $\bar{p}^{3} \mathrm{He}^{+}$, two of them lie within $32 \mathrm{MHz}$ from each other. Therefore these two transitions can be measured with a single cavity with a resonance frequency of $11.14 \mathrm{GHz}$ (and a $\mathrm{Q}$ value of $\sim 100$ ) which is in the middle between the two SSHF transition frequencies. For the other two transitions, another cavity with a resonance frequency of $16.13 \mathrm{GHz}$ has to be used. The whole microwave part of the setup was designed using the High Frequency Structure Simulator (HFSS) Software [14].

\section{Numerical simulations of the transition probabilities}

Due to the limited available measurement time (4-8 weeks) per year, it is particularly important to test the experimental setup and carry out accurate simulations before the actual measurement in order to assess and optimize certain parameters and thus save valuable time. The experiment has been numerically simulated by solving the 
optical Bloch equations. The Bloch equations for a two-level system describe the behaviour of a two-state quantum system in the presence of an oscillatory driving field.

\subsection{Laser transition}

In the case of a laser transition $(n, l)=(36,34)$ to $\left(n^{\prime}, l^{\prime}\right)=(37,33)$, the density matrix for this system leads to the equations

$$
\mathbf{d}\left(\begin{array}{c}
\rho_{p} \\
\rho_{d} \\
\rho_{x} \\
\rho_{y} \\
\rho_{(37,35)} \\
\rho_{(38,34)} \\
\rho_{(38,36)} \\
\rho_{(39,35)}
\end{array}\right)=M\left(\begin{array}{c}
\rho_{p} \\
\rho_{d} \\
\rho_{x} \\
\rho_{y} \\
\rho_{(37,35)} \\
\rho_{(38,34)} \\
\rho_{(38,36)} \\
\rho_{(39,35)}
\end{array}\right)
$$

where

$$
M=\left(\begin{array}{cccccccc}
-\gamma_{r} & 0 & 0 & \frac{1}{2} \Omega_{m}(t) & \gamma_{(37,35)} & 0 & 0 & 0 \\
0 & -\gamma_{A} & 0 & -\frac{1}{2} \Omega_{m}(t) & 0 & \gamma_{(38,34)} & 0 & 0 \\
0 & 0 & -\gamma_{T} & -\Delta \omega & 0 & 0 & 0 & 0 \\
-\Omega_{m}(t) & \Omega_{m}(t) & \Delta \omega & -\gamma_{T} & 0 & 0 & 0 & 0 \\
0 & 0 & 0 & 0 & -\gamma_{(37,35)} & 0 & \gamma_{(38,36)} & 0 \\
0 & 0 & 0 & 0 & 0 & -\gamma_{(38,34)} & 0 & \gamma_{(39,35)} \\
0 & 0 & 0 & 0 & 0 & 0 & -\gamma_{(38,36)} & 0 \\
0 & 0 & 0 & 0 & 0 & 0 & 0 & -\gamma_{(39,35)}
\end{array}\right)
$$

$M$ is the transition matrix, $\rho_{p}$ and $\rho_{d}$ indicate the probabilities of the quantum system to be in the parent state or in the daugther state, $\rho_{x}$ and $\rho_{y}$ give the complex dependency of the transitions for the real and imaginary parts. $\Delta \omega$ denotes the difference between the transition frequency $\omega_{i j}=\frac{E_{i}-E_{j}}{\hbar}$ and the frequency of the oscillating field, with $E_{i}$ and $E_{j}$ being the energies of the two states. $\Omega_{m}(t)$ is the so-called Rabi frequency. For a laser transition this is defined as $\Omega_{m}(t)=\frac{\mu_{m} E(t)}{\hbar}$, $E(t)$ being the time-dependent electric field of the laser radiation. Here $\mu_{m}$ is the transition dipole moment, depending on the quantum number $m$. The radiative decay rates of the parent state and the daughter state are taken into account by the parameters $\gamma_{r_{p}}=\gamma_{r}$ referring to the radiative transition from the metastable state, $(36,34) \longrightarrow(35,33)$ and $\gamma_{r_{d}}=\gamma_{A}$ representing the Auger transition from the unstable state $(37,33)$. The total decay rate can be defined as $\gamma_{T}=\frac{\gamma_{r}+\gamma_{A}}{2}+\gamma_{c_{L}}$ where $\gamma_{c_{L}}$ is the rate for collisions with other helium atoms [15]. The calculated values for these decay rates are $\gamma_{r}=480.7 \mathrm{MHz}$ and $\gamma_{A}=330 \mathrm{MHz}$. The remaining parameters in this matrix take into consideration the refilling from upper states which also has an effect on the population of the measured states - for the parent state these are $\gamma_{(37,35)}$ and $\gamma_{(38,36)}$ and for the daughter state $\gamma_{(38,34)}$ and $\gamma_{(39,35)}$.

\subsection{Microwave transition}

The optical Bloch equations can also be applied to calculate the microwave transition process. These transitions are all between SHF sublevels of the same state $(n, l)=$ 
$(36,34) . \gamma_{r_{p}}=\gamma_{r_{d}}=\gamma_{r}$ is the radiative decay rate from $(36,34) \longrightarrow(35,33), \gamma_{r}=$ 480.7 MHz. The total decay rate $\gamma_{T}$ for the microwave transitions is thus defined as $\gamma_{T}=\gamma_{r}+\gamma_{e}$. In this case $\gamma_{e}$ can be interpreted as the effect of elastic collisions on the dephasing of the coherence between the two SHF states. In a physical situation, there are also inelastic collisions which can induce spin flips between the $J$ states. To take this effect into account, one has to combine the four $4 \times 4$ optical Bloch equations of every transition and the two refilling states to one coupled $18 \times 18$ matrix $M$. The density matrix is given in its complete form in (4). In the magnetic field induced by the microwave, the Rabi frequency is given as $\Omega_{m}(t)=\frac{\mu_{m} B(t)}{\hbar}$ with $B(t)$ denoting the resonating magnetic field. The new parameter $\gamma_{c}$ can be written $\gamma_{c}=\gamma_{r}+3 \gamma_{i}$ where $\gamma_{i}$ is interpreted as the rate of inelastic collisions which induces single spin flip transitions - spin flips of more than one particle are ignored.

The solution to (4) gives-as for the laser transitions-the characteristic Rabi oscillations. For this experiment it is important that the population transfer between two SHF substates induced by the microwave pulse is a maximum. While for the laser transitions it is enough to saturate the transition, in the case of the microwave transition the Rabi oscillations of the magnetic dipole moments are much slower and the states do not decay. The population transfer will be highest after a half Rabi oscillation, a so-called $\pi$-pulse, i.e. when a spin flip occurs. Therefore, the duration of the resonating microwave field and the radio frequency power need to be optimized such that half a Rabi oscillation is induced.

\subsection{Collisional effects}

In order to be able to better understand the line shape of the microwave-induced M1-transitions it is crucial to take into consideration also the collisional effects. The measurement of transitions between the hyperfine structure substates and a comparison to theoretical calculations allows to investigate the collisional processes between the helium atoms of the target medium and the antiprotonic helium atomcules. The elastic and inelastic collisions rates $\gamma_{e}$ and $\gamma_{i}$ can have considerable systematic effects on the signal height, line shape and frequency of the transition line. While inelastic collisions will result in a spin exchange between the hyperfine substates which can lead to a decrease of the measured signal, elastic collisions can cause a broadening and shift of the resonance line. These rates can not be calculated exactly. However, they can both be estimated by comparison of numeric simulations with the experimental results.

It is assumed that initially, at a time $t=0$, SHF substates with different $J$ are all statistically populated. The time dependence of these states will be governed by the same processes as after the first laser-induced depopulation but without microwave field applied. In the case of a simple two-level system the total relaxation rate is the sum of transition rates $\lambda(1 \rightarrow 2)+\lambda(2 \rightarrow 1)$, i.e. approximately twice the rate of the $(2 \rightarrow 1)$ transition. For an eight-level system like $\overline{\mathrm{p}}^{3} \mathrm{He}^{+}$this will be more complex. An estimation can be given by taking twice the rate of a dominant transition. At a temperature $T=6 \mathrm{~K}$ and choosing a transition with a rate of $3 \times 10^{6} \mathrm{~s}^{-1}$, the estimated survival probability for the population of this state after $t=750 \mathrm{~ns}$ is $1 \%$, i.e. very small. As a consequence, the optimal laser delay for the measurement will always have to be a compromise. The time interval needs to be short enough in order that the energy states have not yet returned to thermal equilibrium and 


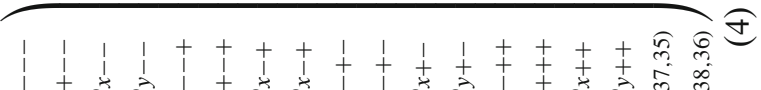

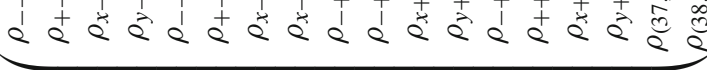

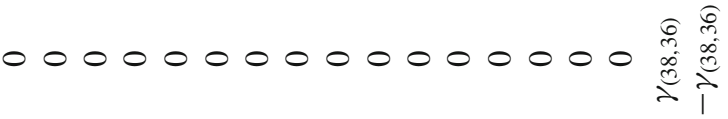

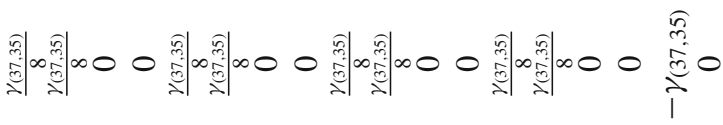

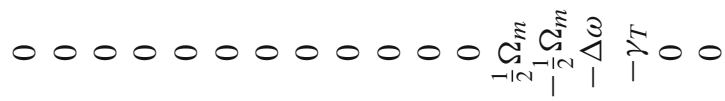

$00000000000000 \frac{1}{3} 00$

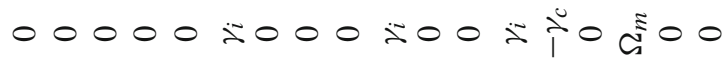

0000 I000

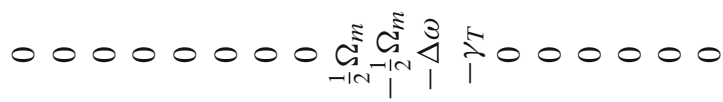

$0000000000 \frac{1}{4} 000000$

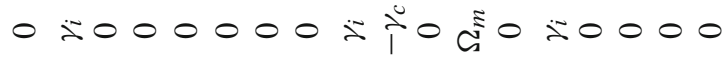

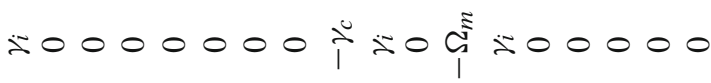

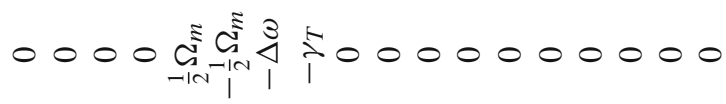

$000000 \$ 30000000000$

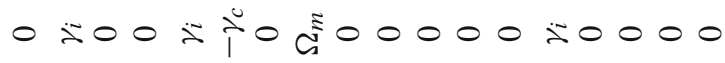

zoo

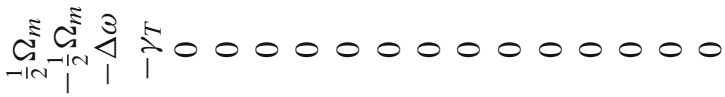

$00 \pm 300000000000000$

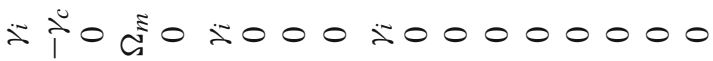

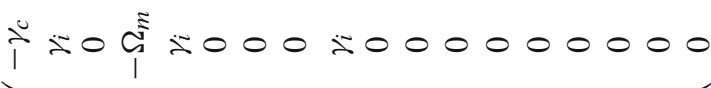

$\|$

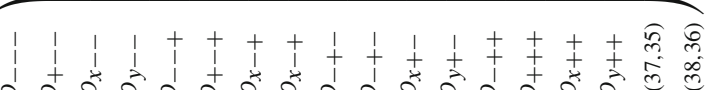

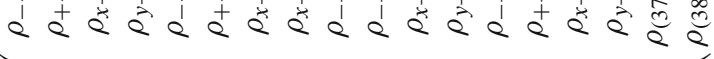


to thus ensure a good population asymmetry between the two SHF substates. However, if the time delay is chosen too short the transition probability will decrease and the line width of the SSHF transition will be broadened. Considering these cicumstances, the optimal delay time between the first and the second laser for our measurements-corresponding to the time the microwave pulse is affecting the population distribution-proved to be about $t=350 \mathrm{~ns}$.

Collision rates To compare the experimental results to the simulated data, the only free parameter in the simulation were the collisional rates, for other decay rates, microwave power, laser delay and cavity characteristics the same values as for the actual measurement were applied. Since the rates of collisional effects can not be calculated exactly, they have to be achieved by comparing the measured data to the simulation. The values thus obtained are similar to those for $\overline{\mathrm{p}}^{4} \mathrm{He}^{+}$. Figure 3 shows the simulated transition lines for the two observed SSHF transitions in comparison to the fitted data. The elastic collisions rate is about $2 \mathrm{MHz}$ and the rate for the inelastic collisions between antiprotonic helium atomcules and helium atoms-which causes an electron spin exchange-is $\sim 1.1 \mathrm{MHz}$. The theoretical estimate for the inelastic collisional relaxation effects is $1 \mathrm{MHz}$ at a temperature of $6 \mathrm{~K}$ [16]. There is no estimation from theory for the elastic collision rate.

\section{Results}

First, a scan over the laser frequency range was done to determine the frequency offset and the splitting of the two HF lines (see Fig. 2) to ensure that only one of the two hyperfine levels of the $(n, L)=(36,34)$ state is depopulated by laser stimulation.

The splitting is $\Delta f=1.72 \pm 0.03 \mathrm{GHz}$, similar to the transition in $\overline{\mathrm{p}}^{4} \mathrm{He}^{+}$, with a splitting of $\Delta f=1.75 \pm 0.01 \mathrm{GHz}$. Due to the different SSHF energy level spacings, one of the laser transition peaks has a lower amplitude and larger width. Each of these peaks consists of another two sub-peaks, corresponding to transitions from one SHF substate of the parent state to the same SHF substate of the daughter state. Two of the four SHF substates, respectively, are lying close enough to each other to have a frequency difference smaller than the laser linewidth $(\sim 100 \mathrm{MHz})$ and the Doppler width $(\sim 300 \mathrm{MHz})$ and can thus not be resolved while the other two lines have a splitting in the range of the broadening and thus result in a smaller and broader peak. The measurements were all performed with a delay time $T$ between the two lasers of $350 \mathrm{~ns}$ and a target pressure of $250 \mathrm{mbar}$.

Two of the four allowed SSHF resonance transitions in $\overline{\mathrm{p}}^{3} \mathrm{He}^{+}$could be observed. The scans of the two microwave-induced transitions are displayed in Fig. 3. They were both fitted with the function of their natural line shape [17].

From the fit, the frequencies for the measured $v_{\mathrm{HF}}^{--}$and $v_{\mathrm{HF}}^{-+}$transitions can be obtained. All relevant results are summarized in Table 1. For a pulse of length $T=$ $350 \mathrm{~ns}$ the expected width is $\Gamma=2.28 \mathrm{MHz}$ [17], which is roughly in agreement with the measurement. In order to determine the optimum power to induce an electron spin flip and thus the maximum population transfer between two SSHF states, the signal was tested at several different microwave powers. The $v_{\mathrm{HF}}^{--}$transition was measured with a power of about $10 \mathrm{~W}$ and the $v_{\mathrm{HF}}^{-+}$transition at about $7.5 \mathrm{~W}$ (for further detail refer to Friedreich et al. [7]). 


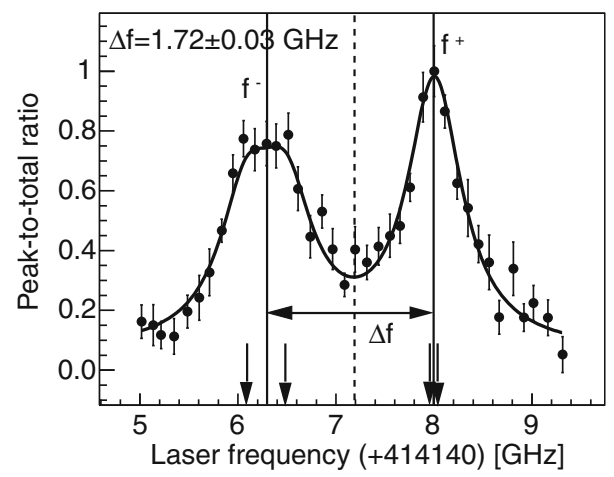

Fig. 2 Laser resonance profile for the $(n, L)=(36,34)$ state of $\overline{\mathrm{p}}^{3} \mathrm{He}^{+}$, displaying the two laser transitions $f^{+}$and $f^{-}$between the HF states of the parent and the daughter state, at a target pressure of $250 \mathrm{mbar}$. The peaks are fitted with four Voigt functions referring to the four allowed E1 transitions between the SHF states of the parent state (Fig. 1). The arrows indicate the corresponding theoretical transition frequencies
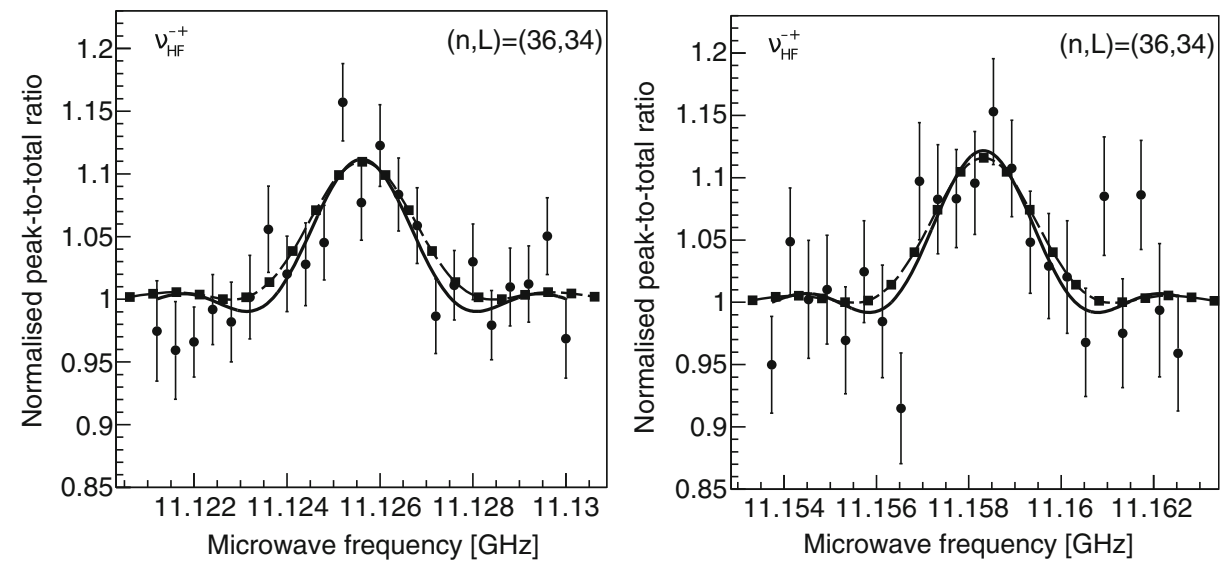

Fig. 3 Scan over the microwave frequency for two of the four SSHF transitions for the $(n, L)=$ $(36,34)$ state of $\bar{p}^{3} \mathrm{He}^{+}$, at a target pressure of $250 \mathrm{mbar}$. Each transition is fitted with the line shape for a pure two-body system (solid lines) [17]. The frequencies of the measured transitions are 11.12559(14) GHz and 11.15839(18) GHz. The dashed curve shows a simulation using collision rates obtained from comparison between experiment and simulation

Table 1 The first experimental results for the $v_{\mathrm{HF}}^{--}$and $v_{\mathrm{HF}}^{-+}$in comparison with three-body QED calculations, where $\nu_{\mathrm{HF}}$ denote the SSHF transition frequencies, $\delta_{\exp }$ is the relative error of the measured frequencies and $\Gamma$ the resonance line width

\begin{tabular}{lcllll}
\hline & $\nu_{\exp }(\mathrm{GHz})$ & $\delta_{\exp }(\mathrm{ppm})$ & $\Gamma(\mathrm{MHz})$ & Korobov $[18,19]$ & $\Delta \nu_{\text {th-exp }}(\mathrm{ppm})$ \\
\hline$\nu_{\mathrm{HF}}^{--}(\mathrm{GHz})$ & $11.12559(14)$ & 13 & $2.08(22)$ & $11.12500(55)$ & -53 \\
$\nu_{\mathrm{HF}}^{-+}(\mathrm{GHz})$ & $11.15839(18)$ & 16 & $1.92(19)$ & $11.15773(55)$ & -59 \\
$\Delta \nu_{\mathrm{HF}}^{ \pm}(\mathrm{GHz})$ & $0.03279(22)$ & & & $0.0327219(16)$ & \\
\hline
\end{tabular}

The theoretical precision is $\sim 5 \times 10^{-5}$. 
The measured hyperfine transition frequencies agree with theory [18] within less than $1 \mathrm{MHz}$. A comparison of the theoretical values for the two SSHF transitions at $11 \mathrm{GHz}$ with the measurement results shows that there is a small shift in frequency towards higher values for both transitions. The frequency difference $\Delta v_{\text {th-exp }}$ between theory and experiment is $\sim 0.6 \mathrm{MHz}$ for $v_{\mathrm{HF}}^{--}$and $\sim 0.7 \mathrm{MHz}$ for $v_{\mathrm{HF}}^{-+}$respectively. The deviation between the experimental and theoretical values for the frequency difference $\Delta v_{\mathrm{HF}}^{ \pm}=v_{\mathrm{HF}}^{-+}-v_{\mathrm{HF}}^{--}$between the two SSHF lines at $11 \mathrm{GHz}$ is $68 \mathrm{kHz}$ out of $32 \mathrm{MHz}$. This difference is important due to its proportionality to the magnetic moment of the antiproton $[7,20]$.

\section{Conclusions}

We have reported on the first microwave spectroscopic measurements of the hyperfine structure of $\overline{\mathrm{p}}^{3} \mathrm{He}^{+}$. Furthermore, the agreement of the transition line shapes with numerical simulations has been demonstrated. The collision rates obtained from a comparison of measured and numerically simulated data, $\gamma_{e}=2 \mathrm{MHz}$ and $\gamma_{i}=1.1 \mathrm{MHz}$, did largely agree with theoretical estimates.

Two of the four favoured SSHF resonance transitions in $\overline{\mathrm{p}}^{3} \mathrm{He}^{+}$were observed and are in accordance with theory [18] within the estimated theoretical error. Also the frequency difference $\Delta v_{\mathrm{HF}}^{ \pm}$agrees with theoretical calculations. However, the experimental error for $\Delta v_{\mathrm{HF}}^{ \pm}$is still very large compared to theory.

A systematic study of these transitions and improved statistics will allow a higher precision, in particular for the frequency difference between the SSHF transition frequencies, for which the experimental error is still considerably larger than the theoretical error. It is further planned to measure also the two SSHF transitions at $16 \mathrm{GHz}$ in order to obtain a complete picture of the $\overline{\mathrm{p}}^{3} \mathrm{He}^{+}$hyperfine structure.

Acknowledgements We express our gratitude to Dr. V. Korobov and Dr. G. Korenman for fruitful discussions on the theoretical framework. We are further grateful to Ms. Silke Federmann and project student Mr. Mario Krenn for their help before and during the beamtime. This work has received funding from the Austrian Science Fund (FWF): [I-198-N20] as a joint FWF-RFBR (Russian Foundation for Basic Research) project, the Austrian Federal Ministry of Science and Research, the Japan Society for the Promotion of Science (JSPS), the Hungarian National Science Funds (OTKA K72172), the European Science Foundation (EURYI) and the Munich Advanced Photonics Cluster (MAP) of the Deutsche Forschungsgemeinschaft (DFG).

Open Access This article is distributed under the terms of the Creative Commons Attribution License which permits any use, distribution, and reproduction in any medium, provided the original author(s) and the source are credited.

\section{References}

1. Iwasaki, M., et al.: Discovery of antiproton trapping by long-lived metastable states in liquid helium. Phys. Rev. Lett. 67, 1246-1249 (1991)

2. Yamazaki, T., et al.: Formation of long-lived gas-phase antiprotonic helium atoms and quenching by $\mathrm{H}_{2}$. Nature 361, 238-240 (1993)

3. Yamazaki, T., et al.: Antiprotonic helium. Phys. Rep. 366, 183-329 (2002)

4. Hayano, R.S., et al.: Antiprotonic helium and CPT invariance. Rep. Prog. Phys. 70(12), 19952065 (2007) 
5. Widmann, E., et al.: Hyperfine structure of antiprotonic helium revealed by a laser-microwavelaser resonance method. Phys. Rev. Lett. 89, 243402 (2002)

6. Pask, T., et al.: A precise measurement of the antiprotonic helium hyperfine structure. Phys. Lett. B 678, 55-59 (2009)

7. Friedreich, S., et al.: First observation of two hyperfine transitions in antiprotonic ${ }^{3} \mathrm{He}$. Phys. Lett. B 700(1), 1-6 (2011)

8. Maury, S., et al.: Status of the antiproton decelerator: AD. Nucl. Phys. B (Proc. Suppl.) 56A, 349-357 (1997)

9. Hori, M., et al.: Analog Cherenkov detectors used in laser spectroscopy experiments on antiprotonic helium. Nucl. Instrum. Methods A 496, 102-122 (2003)

10. Hori, M., et al.: Determination of the antiproton-to-electron mass ratio by precision laser spectroscopy of $\overline{\mathrm{p}} \mathrm{He}^{+}$. Phys. Rev. Lett. 96, 243401 (2006)

11. Pask, T.: A precise measurement of the antiprotonic helium hyperfine structure. $\mathrm{PhD}$ Thesis at the University of Vienna (2008)

12. Sakaguchi, J., et al.: Cryogenic tunable microwave cavity at $13 \mathrm{GHz}$ for hyperfine spectroscopy of antiprotonic helium. Nucl. Instrum. Methods A 533, 598-611 (2004)

13. Massiczek, O., et al.: Liquid helium-free cryostat and hermetically sealed cryogenic microwave cavity for hyperfine spectroscopy of antiprotonic helium. Nucl. Instrum. Methods A 659, 55-60 (2011)

14. Ansoft. http://www.ansoft.com/products/hf/hfss/ (2010)

15. Korenman, G.Y., Yudin, S.N.: Coupled-channel analysis of collisional effects on HFS transitions in antiprotonic helium atoms. J. Phys. B 39(6), 1473-1484 (2006)

16. Korenman, G.Y.: Personal communication (2010)

17. Flugge, S.: Encyclopedia of physics. Springer Verlag, Berlin (1959)

18. Korobov, V.: Hyperfine structure of the metastable states of the ${ }^{3} \mathrm{He}^{+}=\overline{\mathrm{p}}$ atom. Phys. Rev. A 73, 022509 (2006)

19. Korobov, V.I.: Personal communication (2010)

20. Bakalov, D., Widmann, E.: Determining the antiproton magnetic moment from measurements of the hyperfine structure of antiprotonic helium. Phys. Rev. A 76, 012512 (2007) 